\title{
Letter to the editor: Measles on the cruise ship: links with virus spreading into an emergency department in Southern Italy
}

V Cozza ${ }^{1,2}$, M Chironna $^{3}$, C Leo ${ }^{4}$, R Prato (rosa.prato@unifg.it) ${ }^{1}$

1. Department of Medical and Surgical Sciences, University of Foggia, Foggia, Italy

2. European Programme for Intervention Epidemiology Training (EPIET), European Centre for Disease Prevention and Control, $(E C D C)$, Stockholm, Sweden

3. Department of Biomedical Sciences and Human Oncology, University of Bari Aldo Moro, Bari, Italy

4. Prevention Department, Brindisi Local Health Unit, Brindisi, Italy

Cozza V, Chironna M, Leo C, Prato R. Letter to the editor: Measles on the cruise ship: links with virus spreading into an emergency department in Southern Italy. Euro Surveill. 2014;19(19):pii=20800. Available online: http://www.eurosurveillance.org/ViewArticle.aspx?Articleld=20800

Article submitted on 07 May 2014/ published on 15 May 2014

\section{To the Editor:}

Recently, Lanini et al. described an outbreak of measles that occurred on a ship cruising the western Mediterranean Sea, involving 27 cases among crew members and passengers between 20 February and 1 March 2014 [1]. Cases originated from different countries, six were from Italy. As highlighted by the authors, at the time of their preliminary report, the number of cases among passengers was likely to be underestimated, given that the incubation time for measles ranges from seven to 18 days, and the mean passenger time on board is seven days. Since the time on board is not long enough to develop symptoms in a susceptible exposed person, passengers might develop the diseases only after returning to their home country [1].

In the context of these circumstances and the possibility to further spread of measles, we would like to discuss the serious consequences that such an event could have in areas where pools of susceptible persons still exist.

On 27 February 2014, an unimmunised Italian teenager presented to the Emergency Department (ED) of a hospital in Brindisi province, Puglia region, Italy, with fever and rash; the patient was hospitalised and subsequently diagnosed with measles. The strain was confirmed to be identical to the outbreak strain reported by Lanini et al. (MVs/Tonbridge.GBR/5.14). In a letter to the Editor published in Eurosurveillance on 17 April 2014, Mandal et al. hypothesised that the index case of the cruise ship epidemic was likely to be symptomatic between 4 and 10 February 2014 [2], and our epidemiological investigation revealed that, between 6 and 13 February 2014, our patient had indeed been a passenger on the ship where the outbreak occurred.

This was the first measles case reported in Brindisi province since the beginning of 2014. By the end of
April, we observed a sharp increase in the number of cases, developing into a rapidly spreading outbreak that was reaching its fifth generation [3]. As of 8 May 2014, 32 cases have been identified through the enhanced measles surveillance system and active contact tracing; 17 were female, the median age was 19 years (range: $0-39$ years), and 25 were hospitalised. Of 32 cases, one had been vaccinated with one dose of measles-mumps-rubella vaccine (MMR); two cases received one dose as post-exposure prophylaxis during the epidemic.

Initially, there was no evident relationship between most of the affected subjects, but contact tracing by electronic medical records and individual interviews suggested that most cases were associated with the first reported case and, therefore, with the outbreak on the cruise ship. Our epidemic appears to be a secondary outbreak spreading in a hospital ED as a common setting of exposure.

The measles virus strain isolated from the 12 secondary cases that have been typed was indistinguishable from the outbreak strain on the cruise ship. Three of them were attending the ED for a different medical condition at the time when the cruise passenger was there for suspected measles, a time that is compatible with the measles incubation period. The other nine cases belonged to the second (two cases), third (one case), fourth (four cases) and fifth (two cases) generation of infections [3]. With the exception of four small family clusters (range: 2-5 cases), 12 of the 32 secondary cases had been exposed to a measles case in the ED or infectious disease ward of the Brindisi hospital during a time period compatible with their incubation period, either as visitors, patients or healthcare workers.

To contain the outbreak, immediate vaccination of close contacts was carried out, but the hypothesis of 
a common exposure in the hospital setting was formulated late, and adequate control measures such as a separate waiting room for patients with symptoms compatible with measles, were only adopted after 15 April.

Our epidemiological investigation faced similar challenges of measles outbreak management as discussed in the paper by Lanini et al. Similar to a cruise ship, the waiting room of a hospital ED is a closed setting, with constant movement in and out of people with unknown vaccination status; the return to their home countries of passengers incubating the infection is analogous to the return of patients or visitors from the hospital to their own family. Compared with the cruise ship scenario, the risk related to the spread of measles in hospital settings could be even more severe, especially if the vaccine coverage among healthcare workers is low, their attitude towards vaccine uptake is negative [4], and control measures are not implemented early enough.

We believe that the specific characteristics of our outbreak can provide additional input to the measles elimination plan. It highlights the need to consider the risk of measles transmission in EDs, particularly among travellers, with urgent isolation of suspected cases, especially in contexts where vaccination coverage with MMR vaccine is less than $95 \%$ in newborns and lower still in adolescents and young adults. The accidental introduction of measles to healthcare facilities could generate dangerous consequences as in our outbreak where an infant under the age of one year and a woman in the third month of pregnancy were accidentally infected in the ED.

\section{Acknowledgments}

We acknowledge Dr. Maria Giovanna Cappelli who supported us in the epidemiological investigation and Dr. Anna Sallustio for carrying out the microbiological investigation. Prof. Domenico Martinelli gave us valuable advice on contact tracing by medical record-keeping.

\section{Conflict of interest}

None declared.

\section{Authors' contributions}

Vanessa Cozza acted as principal investigator, contributed to the concept and drafted the letter. Maria Chironna supervised the microbiological investigation and the sequencing. Carlo Leo carried out the epidemiological investigation. Rosa Prato acted as outbreak coordinator, contributed to the concept and edited the letter.

\section{References}

1. Lanini S, Capobianchi M, Puro V, Filia A, Del Manso M, Kärki

$T$, et al. Measles outbreak on a cruise ship in the western

Mediterranean, February 2014, preliminary report. Euro

Surveill. 2014;19(10): $\mathrm{pii}=20735$.
2. Mandal S, Ramsay M, Brown K. Measles on a cruise ship: links with the outbreak in the Philippines. Euro Surveill. 2014;19(15): $\mathrm{pii}=20774$.

3. Chiew M, Gidding HF, Dey A, Wood J, Martin N, Davis S, et al. Estimating the measles effective reproduction number in Australia from routine notification data. Bull World Health Organ. 2014;92(3):171-7. http://dx.doi.org/10.2471/ BLT.13.125724

4. Centers for Disease Control and Prevention (CDC). Hospitalassociated measles outbreak - Pennsylvania, March-April 2009. MMWR Morb Mortal Wkly Rep. 2012;61(2):30-2.

5. Caputi G, Tafuri S, Chironna M, Martinelli D, Sallustio A, Falco A, et al. An outbreak of measles including nosocomial transmission in Apulia, south-east Italy, January-March 2008-a preliminary report. Euro Surveill. 2008;13(16):pii=18839. 